\title{
BMJ Open Intrauterine insemination: a UK survey on the adherence to NICE clinical guidelines by fertility clinics
}

\author{
Dongah Kim, ${ }^{1}$ Tim Child, $^{2}$ Cynthia Farquhar ${ }^{1}$
}

To cite: Kim D, Child T, Farquhar C. Intrauterine insemination: a UK survey on the adherence to NICE clinical guidelines by fertility clinics. BMJ Open 2015;5:e007588. doi:10.1136/bmjopen-2015007588

- Prepublication history for this paper is available online. To view these files please visit the journal online (http://dx.doi.org/10.1136/ bmjopen-2015-007588).

Received 6 January 2015 Revised 14 April 2015 Accepted 16 April 2015

\section{CrossMark}

\footnotetext{
${ }^{1}$ Department of Obstetrics and Gynaecology, University of Auckland, Auckland, New Zealand ${ }^{2}$ Oxford Fertility Unit, University of Oxford, Oxford, UK
}

Correspondence to Dr Dongah Kim; dongahk@gmail.com

\begin{abstract}
Objective: To evaluate the awareness and response of fertility clinics in the UK to the National Institute for Health and Care Excellence (NICE) guideline recommendation that intrauterine insemination (IUI) should not be offered routinely, in order to report on current practice in the UK.
\end{abstract}

Design: Online questionnaire survey of fertility clinics in the UK regarding their current clinical practice of IUI, formal discussion of the guideline recommendations, and any alterations made since the recommendations. Setting: 66 UK fertility clinics licensed to provide IUI. Participants: 46 fertility clinics, including 6 clinic groups which represent $70 \%$ of all clinics and clinic groups licensed to provide IUI in April 2014 when the survey email was sent.

Results: Of the 46 clinics that responded, $96 \%$ (44/46) of clinics continue to offer IUI. $98 \%$ (43/44) of those offering IUI also use ovarian stimulation. The most commonly used medications for ovarian stimulation are gonadotrophins (95\%), followed by clomiphene citrate (49\%) and letrozole (19\%). 78\% (36/46) of clinics had formally discussed NICE guideline recommendations. 17 clinics (37\%) had made some changes to their practices; as a result, four clinics reported a reduction in the number of IUI cycles, six clinics had restricted the indications for IUI, and five clinics had begun informing patients of the guideline recommendations, while two did not specify.

Conclusions: The majority of clinics were aware of the guideline recommendations. However, only a small proportion of clinics had made significant changes to their practice by reducing the number of IUI cycles or restricting the clinical indications for IUI. The availability of further evidence will assist NICE and clinicians in making recommendations on the use of IUI. There is a need to further explore the reasons for the lack of adherence to the recommendations.

\section{INTRODUCTION}

Intrauterine insemination (IUI) is a procedure typically used in the treatment of couples with unexplained infertility. It involves the placement of sperm directly into the uterus at the time of ovulation, either in a natural

\section{Strengths and limitations of this study}

- The survey was emailed to all but three of the 85 UK fertility clinics licensed to carry out intrauterine insemination.

- A response rate of $70 \%$ was achieved with the use of an electronic response system and direct communication with the lead clinician or quality manager of each clinic.

- A comparison of some important characteristics showed no major differences between the survey participants and non-responding clinics.

- The timing of the survey was 1 year after the recommendations were published in February 2013. Some of the survey data related in the 2013 year may not have left sufficient time for the changes in practice to impact on the data.

menstrual cycle or following ovarian stimulation. IUI has the advantages of being less invasive and more affordable than other assisted reproduction techniques such as in vitro fertilisation (IVF). However, randomised controlled trials of IUI with and without stimulation compared with no treatment have not provided convincing evidence of effectiveness of the procedure. ${ }^{1} 2$ Pregnancy rates have been observed to be higher when IUI is combined with ovarian stimulation, however, this has to be weighed against the risks of ovarian hyperstimulation and multiple pregnancies. ${ }^{3}$

The latest National Institute for Health and Care Excellence (NICE) guideline on fertility (2013) recommends that IUI should not be routinely offered for people with unexplained infertility, mild endometriosis or 'mild male factor infertility' who are having regular unprotected sexual intercourse. ${ }^{4}$ This is referring to the treatment either with or without ovarian stimulation, and acknowledges that exceptional circumstances include, for example, when people have social, cultural or religious objections to IVF. Instead, the guidelines recommend considering IVF as first-line treatment after 
2 years of expectant management. The recommendation was informed by two randomised controlled trials of IUI that did not find evidence of benefit when compared with expectant management. ${ }^{12}$ A recent survey of fertility doctors in the UK with a response rate of $33 \%$ found that $39 \%$ of respondents agreed with the recommendation that all patients with unexplained subfertility should be offered IVF directly, and $27 \%$ reported that they would change their treatment strategy. ${ }^{5}$

This study hypothesised that the adherence to the recent NICE guideline recommendations would reflect the ongoing controversy surrounding the use of IUI as a treatment for infertility. The objective of this questionnaire survey was to evaluate the awareness and response of fertility clinics to the recommendation regarding IUI, in order to report on current practice in the UK.

\section{METHOD}

An online questionnaire survey was used as it was simple and easy to distribute and complete. The questionnaire was designed using an online survey software tool, Survey Monkey. All clinics licensed to carry out IUI were identified using an advanced clinic search with the keyword 'insemination' on the Human Fertilisation and Embryology Authority (HFEA) website. The clinic email address was obtained from either the HFEA website, the clinic's website, or the clinic was directly rung when no email address was acquired from these two locations. Of the 85 clinics identified, 82 clinics were contacted by email (three clinics had no email addresses available and were not contactable by phone on numerous occasions). Some of these clinics were noted to be of the same clinic group. Eight such clinic groups were identified through clinic website searches. Although an email was sent to each of the clinics, the answers were analysed for consistency prior to a clinic group being collated as one for the purposes of this study. At the end of the study period, a total of 66 clinics/clinic groups were approached by email.

A link to the survey was emailed with an explanatory cover letter. The clinics were requested to identify themselves solely for the purpose of knowing which clinics had not completed the survey, although it was emphasised that all findings would be anonymised. The questionnaire comprised seven short multiple choice questions with a section for additional comments. The questionnaire was revised by three fertility consultants and focused on whether the clinics were offering IUI, with or without ovarian stimulation, whether the guidelines have been discussed at a clinic meeting, and if any changes had been made to clinical practice. Ethical consent was not required for this survey as the findings were kept anonymous and there was a link to opt out of the survey. The survey was conducted from 8 April 2014, with an email reminder sent out a week later, and further links sent to alternative emails in the remaining non-responders. The last of the completed surveys was received on 2 June 2014. The clinic data available on the HFEA website was used to assess for potential differences between the participants and nonresponding clinics (table 2).

The data was anonymised and analysed on Excel. Descriptive statistics were calculated only. A search was carried out on the HFEA website for the success rate of IUI with partner sperm for each clinic to obtain the figures for the IUI cycles in 2013. The data was obtained for 61 clinics, including the three clinics not contactable for the survey. The data were not available for eight clinics. Eight clinics had data available for 2013, but are no longer licensed to provide IUI.

\section{RESULTS}

Forty-six of $66(70 \%)$ clinics, or clinic groups, responded to the survey. There were a total of 58 responses. Seven clinics provided more than one response, and there were five responses from within the same clinic groups. Four of the six clinic group participants had more than one clinic respond to the survey. These responses were found to have generally consistent answers prior to being collated into clinic groups. Two clinic groups (one group consisting of two clinics and the other of three clinics) had only one clinic respond, in which case their answers were taken as representative of the clinic group. Responses were received from fertility clinics in England, Scotland, Northern Ireland and Wales. Of the 82 individual clinics contacted by email, responses were received from all the three clinics in Wales, one of the three clinics in Northern Ireland, three of the five clinics in Scotland and 44 of the 71 clinics in England. Three clinic groups comprise clinics in more than one country.

The key findings are shown in table 1 . The survey findings indicate that $96 \%(44 / 46)$ of clinics are continuing to offer IUI. Ninety-eight per cent $(43 / 44)$ of those offering IUI use ovarian stimulation. The most commonly used medications for ovarian stimulation are gonadotrophins $(95 \%)$, followed by clomiphene citrate $(49 \%)$ and letrozole $(19 \%)$.

Seventy-eight per cent $(36 / 46)$ of clinics had discussed NICE guideline recommendations. The remaining clinics had either not considered the recommendations (4), did not respond (2), did not know (2) or did not offer IUI (2). Seventeen clinics (37\%) had made changes to their practices; four clinics reported a reduction in the number of IUI cycles, six clinics had restricted the indications for IUI, and five clinics had begun informing patients of the guideline recommendations, and two did not specify. Of the 25 clinics that had not made any changes, 2 were offering the treatment previously and had since stopped, and 4 had already made restrictions on the indications for IUI.

The clinical indications for IUI cycles in 2013 were unexplained infertility at 26 clinics, endometriosis at 10 clinics, male factor infertility at 13 clinics, and all 3 indications at 9 clinics. Ten clinics did not answer the question. Two clinics noted that the IUI cycles in 2013 were only offered 
Table 1 Survey responses from fertility clinics in the UK

\begin{tabular}{|c|c|c|c|c|c|}
\hline Question & Total (\%) & Yes (\%) & No (\%) & $\begin{array}{l}\text { Don't } \\
\text { know (\%) }\end{array}$ & $\begin{array}{l}\text { Did not } \\
\text { answer (\%) }\end{array}$ \\
\hline 1. Is your clinic offering intrauterine insemination? & 46 & $44 / 46(96)$ & $2 / 46(4)^{*}$ & $0(0)$ & $0(0)$ \\
\hline $\begin{array}{l}\text { 2. If yes, are you offering intrauterine insemination with } \\
\text { ovarian stimulation? }\end{array}$ & 44 & $43 / 44(98)$ & $0(0)$ & $0(0)$ & $1 / 44(2)$ \\
\hline \multicolumn{6}{|l|}{ 3. If yes, which of the following medications are used? $\dagger$} \\
\hline Gonadotropins & 43 & $41 / 43$ & $1 / 43$ & 0 & $1 / 43$ \\
\hline Clomiphene citrate & 43 & $21 / 43$ & $21 / 43$ & 0 & $1 / 43$ \\
\hline Letrozole & 43 & $8 / 43$ & $34 / 43$ & 0 & $1 / 43$ \\
\hline $\begin{array}{l}\text { 4. Has the } 2013 \text { NICE clinical guideline } 156 \text { been discussed } \\
\text { at a clinic meeting? } ¥\end{array}$ & 46 & $36 / 46(78)$ & $6 / 46(13)$ & $2 / 46(4)$ & $2 / 46(4)$ \\
\hline $\begin{array}{l}\text { 5. Have any alterations in your clinic practice been made } \\
\text { since this recommendation?§ }\end{array}$ & 46 & $17 / 46(37)$ & 25/46 (54) & $2 / 46(4)$ & 2/46 (4) \\
\hline \multicolumn{6}{|c|}{ 6. What were the clinical indications for the intrauterine insemination cycles in $2013 ? \rrbracket$} \\
\hline Unexplained infertility & \multicolumn{5}{|l|}{$26 / 44$} \\
\hline Endometriosis & \multicolumn{5}{|l|}{$10 / 44$} \\
\hline Male factor infertility & \multicolumn{5}{|l|}{$13 / 44$} \\
\hline Cervical factor infertility & \multicolumn{5}{|l|}{$6 / 44$} \\
\hline Immunological factor infertility & \multicolumn{5}{|l|}{$1 / 44$} \\
\hline Only for religious, social, cultural reasons & \multicolumn{5}{|l|}{$2 / 44$} \\
\hline Only for donor insemination and cancer patients & \multicolumn{5}{|l|}{$2 / 44$} \\
\hline Question not answered & \multicolumn{5}{|l|}{$10 / 44$} \\
\hline 7. Table 4 & & & & & \\
\hline
\end{tabular}

for religious, social or cultural reasons, and two clinics offered IUI only for donor insemination and in the treatment of patients with cancer. A range of other indications for IUI were noted by the respondents and included couples with psycho-sexual dysfunction, women with polycystic ovarian syndrome, couples who required donor sperm, women with immunological factors and cervical factors, and couples who wished to avoid IVF.

Table 2 reports on differences between the responding clinics and the non-responding clinics, and there were no significant differences detected.

Table 3 reports on the categories for the number of IUI cycles in each clinic. The majority of clinics were doing less than 100 cycles of IUI per year, and only $34 \%$ of clinics (of those where the data was available) were doing more than 100 IUI cycles per year.
Table 4 shows the figures for the number of IUI cycles with partner sperm undertaken in 2013, and compares the data available on the HFEA website with the data available from clinics that answered the specific question. The HFEA data show that of the 5934 cycles of IUI undertaken in 2013, 4970 cycles $(84 \%)$ were stimulated. With the inclusion of the eight clinics that are no longer licensed to carry out IUI, of the 6944 cycles of IUI undertaken in 2013,5950 cycles $(86 \%)$ were stimulated.

\section{DISCUSSION}

The key finding of this survey is that while the majority of clinics was aware of the recent guideline recommendations, only a small proportion of clinics had made significant changes to their practice by reducing the

Table 2 Comparison of characteristics of responding and non-responding clinics

\begin{tabular}{lccc}
\hline Characteristic & Responding clinics & Non-responding clinics & Total \\
\hline Type of patients, $n(\%)^{*}$ & & & $10(15)$ \\
Private patients only & $6(13)$ & $4(20)$ & $48(73)$ \\
NHS and private & $37(80)$ & $11(55)$ & $8(12)$ \\
NHS patients only & $3(7)$ & $5(25)$ & 66 \\
Total & 46 & 20 & $2(10)$ \\
Clinic groups & $6(13)$ & 8 \\
\hline
\end{tabular}

${ }^{*}(\%)$ is the percentage of the total number of responding clinics, non-responding clinics or total. Percentages do not necessarily sum up to $100 \%$ due to rounding.

NHS, National Health Service. 
Table 3 The total number of women who began intrauterine insemination (IUI) cycles in each clinic in 2013

Total number of IUI
cycles in each clinic
in 2013

Number of
responding
clinics*

Number of non-responding clinics*

\begin{tabular}{lrl}
\hline 0 & 1 & 0 \\
$1-50$ & 17 & 8 \\
$51-100$ & 6 & 6 \\
$101-150$ & 4 & 1 \\
$151-200$ & 3 & 1 \\
$201-250$ & 5 & 1 \\
$251-300$ & 3 & 1 \\
$\geq 301$ & 1 & 0 \\
Only DI data or no data & 6 & 2 \\
available & & \\
Total & 46 & 20 \\
\hline
\end{tabular}

${ }^{*}$ The figures indicate the number of clinics that began IUI cycles for the number of women shown. Source: HFEA Website 2013 (Human Fertilisation and Embryology Authority).

number of IUI cycles or restricting the clinical indications for IUI. Several reasons as to why some clinics have not made changes were given. Several clinics commented that there is continued funding for IUI by local Clinical Commissioning Groups (CCGs). Interestingly, one respondent commented that their own audit findings showed a success rate of up to $28 \%$ with IUI in couples where the clinical indications included unexplained infertility, male factor infertility and endometriosis. Another respondent commented that they disagreed with the guidelines, questioning the evidence behind the recommendations. It was also noted that some clinics were still offering IUI if it was preferred by patients, particularly for self-funded patients, or for patients who have only one cycle of IVF funded by National Health Service (NHS). The majority of responding clinics treat both NHS and private patients, and only 3 of the 46 respondents treat only NHS patients. Private patients may be discouraged from paying for the more expensive IVF treatment, yet on the other hand it may be more financially desirable for clinicians to offer IVF. Guideline adherence may also be affected by whether clinics offer only IUI or both IUI and IVF. Six of the 46 responding clinics offer only IUI, two clinics were previously licensed to carry out IUI, but now only offer IVF, and the remaining 38 clinics offer both IUI and IVF, hence this is unlikely to be of significance.

\section{Strengths and limitations of the survey}

The survey was emailed to all but three of the fertility clinics licensed to carry out IUI in the UK. The response rate was $70 \%$, which is better than other similar surveys exploring guideline awareness and adherence to recommendations. A similar recently published survey focused on the management of women with unexplained subfertility, and also included whether doctors would change practice according to NICE guidelines. ${ }^{5}$ A total of 420 members of the British Fertility Society were contacted by email, and a disappointing response rate of $33 \%$ was reported. Another UK survey conducted in 2012 assessed the management of infertility in women aged 40 years and over. ${ }^{6}$ Sixty-nine fertility clinics offering IVF and IUI were contacted by email, and a better response rate of $64 \%$ was achieved. ${ }^{6}$ A postal survey evaluated clinician attitudes to IUI and was sent to 150 fertility clinics in the $\mathrm{UK}^{7} \mathrm{~A}$ response was received from 101 practitioners; the survey did not specify whether the responses were from different clinics. The better response rate in our own survey may have been due to the use of an electronic response system, and because we communicated directly with the lead clinician or quality manager of each clinic.

It is expected that there would be non-responders to the survey, however, the response rate of 46 of the 66 clinics emailed $(70 \%)$ was sufficient to draw conclusions. It is possible that those who responded were the motivated clinics, in which case the findings would be an overestimation of the actual awareness and adherence to the guidelines. However, a comparison of some important characteristics showing no major differences between the survey participants and non-responding

Table 4 The number of intrauterine insemination (IUI) cycles with partner sperm in the UK during 2013

\begin{tabular}{lll}
\hline $\mathbf{2 0 1 3}$ IUI figures & Clinics who answered Question 7 & HFEA data \\
\hline Total number of cycles & 2146 (24 clinics) & $5934(61$ clinics $)$ \\
Total number of stimulated cycles & 1830 (24 clinics) & $4970(61$ clinics) \\
Total number of unstimulated cycles & 316 (23 clinics) & $964(61$ clinics) \\
$\begin{array}{l}\text { Clinical pregnancy rate } \\
\text { Per stimulated cycle of IUI }\end{array}$ & Median 12\%, range (0-28\%) \\
$\quad$ Per natural cycle of IUI & Median 8.5\%, range (0-100\%) & Median 10.6\%, range (0-24.2\%) \\
\hline
\end{tabular}

Question 7: Finally, if you have access to the following statistics please answer the following:

A. How many women at your clinic underwent intrauterine insemination in 2013?

B. What was the number of stimulated IUI cycles in 2013 ?

C. What was the number of natural IUI cycles in 2013 ?

D. What was the clinical pregnancy rate per stimulated cycle of IUI in 2013?

E. What was the clinical pregnancy rate per natural cycle of IUI in 2013?

HFEA data source: HFEA website 2013 (Human Fertilisation and Embryology Authority). 
clinics is reassuring that the clinics which responded are representative (table 2).

Another potential form of response bias is that the responders may have selected what they believed to be 'right' answers, which would also lead to an overestimation. The anonymity of the findings was emphasised in the cover letter and survey in an attempt to minimise this. The cover letter specifically requested for the email to be forwarded to the most appropriate person, however, it can only be inferred that the personnel from each clinic who completed the survey were knowledgeable about the actual practice in their clinic, aware of whether discussions took place, and of any changes made. It is assumed that the practice protocols and any changes are generally followed through by the consultants within each clinic. In the two clinic groups where there was only one response, it is assumed that the answers are representative of the practice within the clinic groups. A comparison between answers within the other clinic groups (the largest consisting of six clinics where three clinics responded) showed general consistency, however, it is still possible that they are not reflective of practice in all the clinics within a group. If this assumption is inaccurate it would have a relatively minor effect on the findings considering the small total number of clinic groups.

The timing of the survey was 1 year after the recommendations were published in February 2013. Some of the survey data related in the 2013 year may not have left sufficient time for the changes in practice to impact on the data. However, the majority of the survey questions were not related to the 2013 year but more focused on the practice changes made. Furthermore, the survey was conducted between April and June 2014, and adherence to the guidelines since then may have improved due to reduced funding for IUI by CCGs. The survey findings are generalisable to fertility clinics throughout the UK. NICE guidelines recommend the level of funding for fertility treatment in England and Wales.

\section{Relationship between evidence and recommendations}

NICE guideline recommendations were based on studies that showed no evidence of a benefit in terms of live birth rates with IUI compared with expectant management. ${ }^{1}{ }^{2}$ However, the Steures et al study (2006) involved couples with mild unexplained infertility with a probability of pregnancy greater than $30 \%$. The Bhattacharya et $a l^{2}$ study (2008) showed no improvement in live birth rates with unstimulated IUI in couples with unexplained infertility. It has been previously suggested that the success rate of IUI remains controversial due to the influence of multiple factors. ${ }^{7}$ These include the timing, localisation, preparation of sperm, use of ovarian stimulation, the number and size of the follicles and techniques used when carrying out the procedure. Considering the lack of robust evidence to support the guidelines and the range of factors that influence the efficacy of IUI, it is not surprising that the survey findings indicate a variety of different attitudes towards the use of IUI.

Some clinics noted that they are still discussing and offering IUI due to patient preference over IVF. The European Society of Human Reproduction and Embryology Capri Workshop in December 2009 suggested that for couples with a better chance of conception, it is more likely that ovulation induction is equally effective to IUI. For couples with lower chances of conception, IVF would more likely be effective over IUI. They concluded that although IUI may be more affordable and less demanding, IVF is more effective in the treatment of infertility. ${ }^{8}$ This is supported by the findings of the recent FORT-T trial (the Forty and Over Treatment Trial), which compared two cycles of IUI with controlled ovarian stimulation using clomiphene citrate or follicle stimulating hormone (FSH) versus immediate IVF (2014). ${ }^{9}$ This randomised controlled trial involved 154 older couples (women aged between 38 and 42 years) with unexplained infertility and a reasonable chance of conception. In these couples, immediate treatment with IVF was the most efficient option with higher live birth rates and fewer treatment cycles. The previous Fast Track and Standard Treatment trial had suggested that for young couples with unexplained infertility, proceeding to IVF after three cycles of IUI with clomiphene citrate was more cost effective than continuing with further three cycles of IUI with FSH (2010). ${ }^{10}$ However, these findings do not agree with a recently published RCT (INeS) comparing three cycles of IVF with single embryo transfer, and with six cycles of IUI with stimulation which did not report a difference in live birth rate between the two arms of the study. ${ }^{11}$

In addition to the issue of efficacy, the survey respondents highlighted affordability and funding for IVF as influencing clinical practice and guideline adherence. IUI is considerably cheaper than IVF, and if it has similar outcomes then it may be difficult to convince couples to have IVF.

\section{CONCLUSION}

The purpose of NICE guidelines is to optimise the quality of care by assisting clinicians and patients in making appropriate and safe decisions. This survey reflects the continued variation in the practice of IUI in the UK, and a general poor response to the guidelines. There is a need for more high-quality trials such as the FORT-T trial, INeS trial ${ }^{11}$ and the ongoing TUI study. ${ }^{12}$ The availability of further evidence will aid NICE and clinicians in making recommendations on the use of IUI as a treatment for infertility. Adherence to the guidelines will also improve when there is a reflection of the recommendations in the funding by the local CCGs. Further studies that evaluate the specific reasons and barriers to adherence to the guideline will also help. 
Acknowledgements The authors would like to thank all the individuals who took the time to complete the survey. Other individuals who provided invaluable assistance are: Ursula Foley, University of Auckland; Karen Kumar, Oxford Fertility Unit; Andrew Bond, Oxford Fertility Unit; and Clare Lewis-Jones, Infertility Network UK.

Contributors CF and TC were involved in the conception and design of the study. DK and TC were involved in data acquisition. DK and CF carried out data analysis and interpretation. All three authors revised the work.

Funding This research received no specific grant from any funding agency in the public, commercial or not-for-profit sectors.

Competing interests All authors have completed the ICMJE uniform disclosure form and declare no support from any organisation for the submitted work. TC is a member of the NICE Guideline Development Group and the Medical Director of Oxford Fertility Unit, a UK fertility clinic offering both IUI and IVF.

Provenance and peer review Not commissioned; externally peer reviewed.

Data sharing statement Additional data can be accessed via the Dryad data repository at http://datadryad.org/ with the doi:10.5061/dryad.gg47h

Open Access This is an Open Access article distributed in accordance with the Creative Commons Attribution Non Commercial (CC BY-NC 4.0) license, which permits others to distribute, remix, adapt, build upon this work noncommercially, and license their derivative works on different terms, provided the original work is properly cited and the use is non-commercial. See: http:// creativecommons.org/licenses/by-nc/4.0/

\section{REFERENCES}

1. Steures $P$, Van der Steeg JW, Hompes PG, et al. Intrauterine insemination with controlled ovarian hyperstimulation versus expectant management for couples with unexplained subfertility and an intermediate prognosis: a randomised clinical trial. Lancet 2006;368:216-21.

2. Bhattacharya S, Harrild K, Mollison J, et al. Clomifene citrate or unstimulated intrauterine insemination compared with expectant management for unexplained subfertility: pragmatic randomised controlled trial. BMJ 2008;337:a716.

3. Veltman-Verhulst SM, Cohlen BJ, Hughes E, et al. Intra-uterine insemination for unexplained subfertility. Cochrane Database Syst Rev 2012;(4):CD001838.

4. NICE. Nice guideline: Fertility: for people with fertility problems. 2013. NICE clinical guideline 156 February 2013, 1.8.1.3-4.

5. Nandi A, Gudi A, Shah A, et al. An online survey of specialists' opinion on first line management options for unexplained subfertility. Hum Fertil 2015;18:48-53.

6. Marinakis G, Nikolaou D. National survey of the current management of infertility in women aged 40 and over in the UK. J Obstet Gynaecol 2012;32:375-8.

7. Rawal N, Drakeley A, Haddad N. Intrauterine insemination practice in the UK. J Obstet Gynaecol 2008;28:738-41.

8. ESHRE Capri Workshop Group. Intrauterine insemination discussed by the ESHRE Capri Workshop Group 2009. http://www.eshre.eu/ (accessed 30 Aug 2014)

9. Goldman $\mathrm{MB}$, Thornton $\mathrm{KL}$, Ryley $\mathrm{D}$, et al. A randomised clinical tria to determine optimal infertility treatment in older couples: the Forty and Over Treatment Trial (FORT- T). Fert Steril 2014;101:1574-81.

10. Reindollar $\mathrm{RH}$, Regan MM, Neumann PJ, et al. A randomized clinical trial to evaluate optimal treatment for unexplained subfertility: the fast track and standard treatment (FASTT) trial. Fert Steril 2010;94:888-99.

11. Bensdorp AJ, Tjon-Kon-Fat RI, Bossuyt PMM, et al. Prevention of multiple pregnancies in couples with unexplained or mild male subfertility: randomised controlled trial of in vitro fertilisation with single embryo transfer or in vitro fertilisation in modified natural cycle compared with intrauterine insemination with controlled ovarian hyperstimulation. BMJ 2015;350:g7771.

12. Liu E, Armstrong S, Farquhar C. The intrauterine insemination with stimulation in women with unexplained infertility study. ACTRN12612001025820. Date registered 24/09/2012. 